\title{
TÉCNICAS DE INTELIGÊNCIA ARTIFICIAL APLICADAS AO DIREITO: REPRESENTAÇÃO DE CONHECIMENTO E RACIOCÍNIO AUTOMATIZADO
}

\author{
Alexandre Zamberlan'; Robertson E. dos Santos ${ }^{2}$; Darci Ribeiro \\ Francielle A. Tybusch ${ }^{4}$; Márcia A. Bühring ${ }^{5}$; Márcio de S. Bernardes ${ }^{6}$; \\ Paulo J. T. dos Santos ${ }^{7}$; Cristiano Becker ${ }^{8}$
}

\section{RESUMO}

Há crescente discussão no campo do Direito sobre o uso da Inteligência Artificial (IA), seja em processos de automatização de tarefas, classificação de textos, predição de tendência de fundamentação, seja, até mesmo, em processos de tomada de decisão. Ressalta-se que as discussões são guiadas por profissionais da área Jurídica com apoio de profissionais da Ciência da Computação, mas tendem a focar no porquê e no objetivo do uso da Inteligência Artificial. Dessa forma, este trabalho busca mostrar algumas das técnicas da IA e como elas operacionalmente executariam em sistema computadorizado disponível na Internet. Para isso, a metodologia deste estudo é baseada em revisão bibliográfica amparada por um estudo de caso, que busca modelar a dinâmica de um possível sistema com uso de representação de conhecimento e raciocínio automatizado via Ontologia.

Palavras-chave: Descoberta de Conhecimento; Reasoner; Sistema de Comportamento Inteligente; Modelo Conceitual.

Eixo Temático: Tecnologia, Inovação e Desenvolvimento Sustentável (TIDS).

\section{INTRODUÇÃO}

Vive-se um momento ímpar na sociedade dita tecnológica, com inúmeras facilidades e oportunidades, mas também com desafios e situações que merecem discussões mais atentas e pontuais. A tão badalada Inteligência Artificial, ou

\footnotetext{
${ }^{1}$ Autor/Apresentador - Ciência da Computação - UFN - alexz@ufn.edu.br.

${ }^{2}$ Autor - ER Sistemas - robertson@ersistemas.info

${ }^{3}$ Autor - Direito - UFN - darci.ribeiro@ufn.edu.br

${ }^{4}$ Autor - Direito - UFN - francielle.agne@ufn.edu.br

${ }^{5}$ Autor - Direito - UFN - marcia.andrea@ufn.edu.br

${ }^{6}$ Autor - Direito - UFN - marciodesouza@ufn.edu.br

${ }^{7}$ Autor - Direito - UNOESC - pjtrindades@hotmail.com

${ }^{8}$ Autor - Direito - UFN - cbisaia@ufn.edu.br
} 
simplesmente IA, aparece como uma expressão que está no arsenal de empresas, no universo de jogos digitais, em filmes e seriados, e na área Jurídica.

Inteligência Artificial é algo profundo e que envolve outras áreas do conhecimento, como Matemática, Psicologia, Sociologia, Letras (linguística) e Computação (engenharia de software, processamento de imagens e computação de alto desempenho). De fato, a Inteligência Artificial possui técnicas para construção de sistemas com comportamento inteligente, com autonomia, adaptação, iniciativa e capacidade de interação por comunicação falada ou escrita. Esses sistemas aprendem por repetição e buscam reconhecer padrões em imagens, em textos, perfis de redes sociais e tendências em sites de compras, por exemplo. $E$, certamente, existem não só para auxiliar pessoas em suas tarefas diárias, mas também para potencializar negócios de grandes empresas.

Especificamente, um sistema com comportamento inteligente poderia coletar e tratar dados referentes a: i) quais foram as decisões sobre o tema Direito Ambiental nos tribunais; ii) qual padrão seguido ou utilizado por esses tribunais, nas essas decisões iii) quais outras decisões, leis ou autores invocam em suas fundamentações iv) qual a questão ambiental (desastres ou sustentabilidade) que usualmente mais enfrentam? v) quais as tentativas de conciliação ou mediação (ou qualquer outra forma de solução) que antecede a judicialização restou exitosa e, não havendo êxito, quais razões.

Para isso, o sistema inteligente deve ser composto por um modelo conceitual, da área de Direito Ambiental, conhecido como Ontologia, o qual deve representar o conhecimento e, logo, inferir (deliberar) automaticamente sobre essa representação. Os conceitos desse modelo devem ser elencados e relacionados por especialistas da área. Dessa forma, a ontologia deve especificar todos os conceitos que sejam consensuais por especialistas, que possam ser facilmente compartilhados (em geral via Internet) e que possam ser processados por computadores ou dispositivos (GRUBER, 1992). 
Assim, este trabalho traz uma revisão geral sobre as subáreas da Inteligência Artificial e uma discussão de como utilizá-las em alguns contextos, como a área Jurídica. Além disso, apresenta uma modelagem inicial da dinâmica de um possível sistema com o uso de representação de conhecimento e raciocínio automatizado via Ontologia.

\section{REVISÃO BIBLIOGRÁFICA}

A área da Ciência da Computação, certamente, promove a integração com outras áreas de conhecimento, sempre com o propósito de ser o meio ou o recurso facilitador para essas áreas, justamente por possuir algoritmos (conjunto de passos ou procedimentos lógicos bem definidos que levam à solução de um problema), metodologias de projeto e/ou desenvolvimento de sistemas, ferramentas computacionais (software e hardware), linguagens de programação de dispositivos eletrônicos, como ilustra a Figura 1.

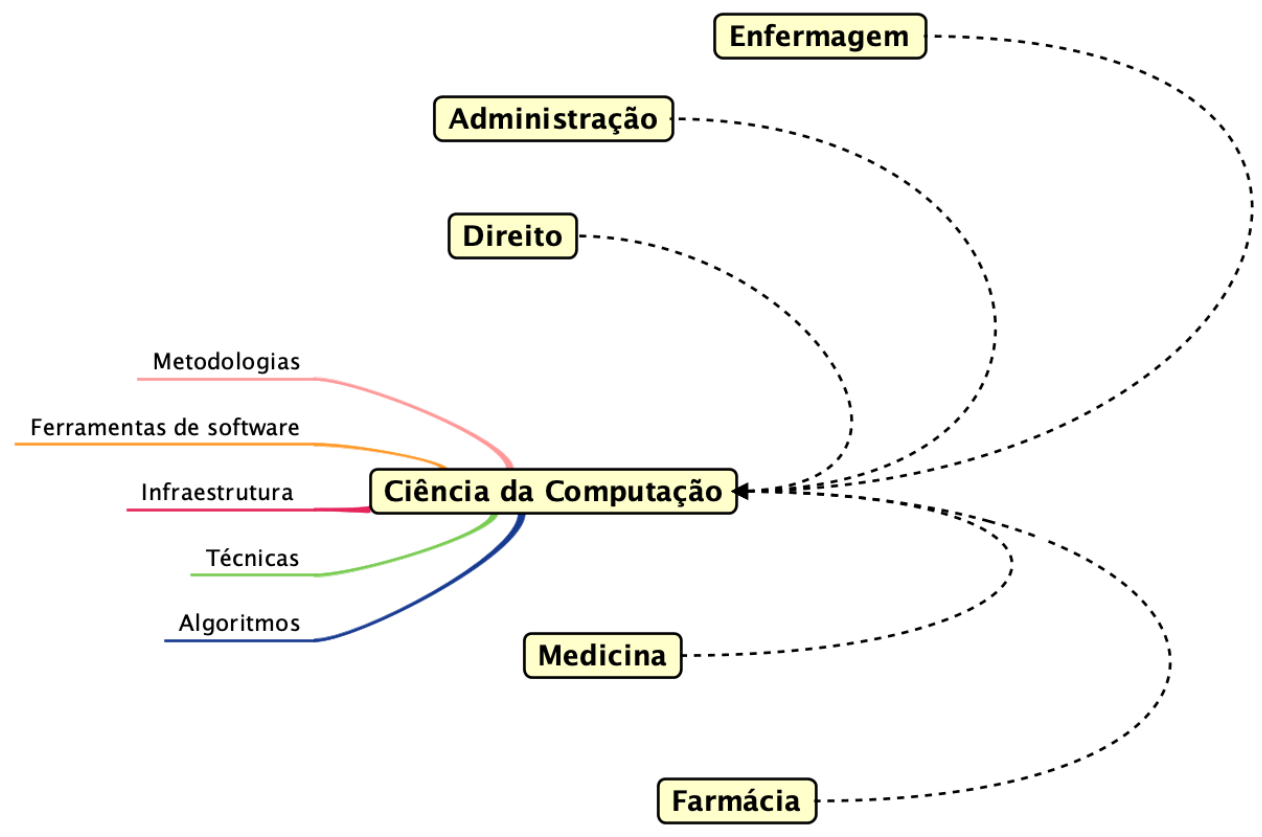

Figura 1 - Mapa mental da relação da Ciência da Computação com algumas áreas.

Em especial, a Inteligência Artificial (IA) tem sido uma das subáreas da Ciência da Computação que mais tem sido comentada, empregada e promovida nas diversas soluções computacionais, como em sistemas administrativos, comerciais, 
industriais, jogos eletrônicos, redes sociais (relacionamento), entre outros. A aplicação de IA nessas áreas de conhecimento pode ser vista como um campo de estudo em constante transformação e essa evolução só acontece devido ao incremento dos recursos computacionais, como memória e processador dos dispositivos (smartphones, computadores, etc.) que executam esses sistemas.

A Figura 2 mostra o papel da IA na resolução de problemas, que em geral deveriam ser resolvidos por especialistas humanos, mas que estão sendo resolvidos por sistemas informatizados projetados e construídos com as técnicas de Processamento da Língua Natural (capacidade de reconhecer elementos da fala e da escrita humana), Métodos de Busca (algoritmos para raciocínios dedutivos e/ou indutivos), Sistemas Multiagentes (técnica para construção de sistemas autônomos, proativos, adaptáveis e com habilidade de comunicação entre seus elementos), Ontologia (capacidade de representação de conhecimento e de raciocínio automatizado), Mineração de Dados (algoritmos para descoberta de conhecimento oculto em base de dados) e Redes Neurais (algoritmos para aprendizagem de máquina, via treinamento repetitivo, para reconhecimento de padrões em base de dados) (NORVIG; RUSSEL, 2014) .

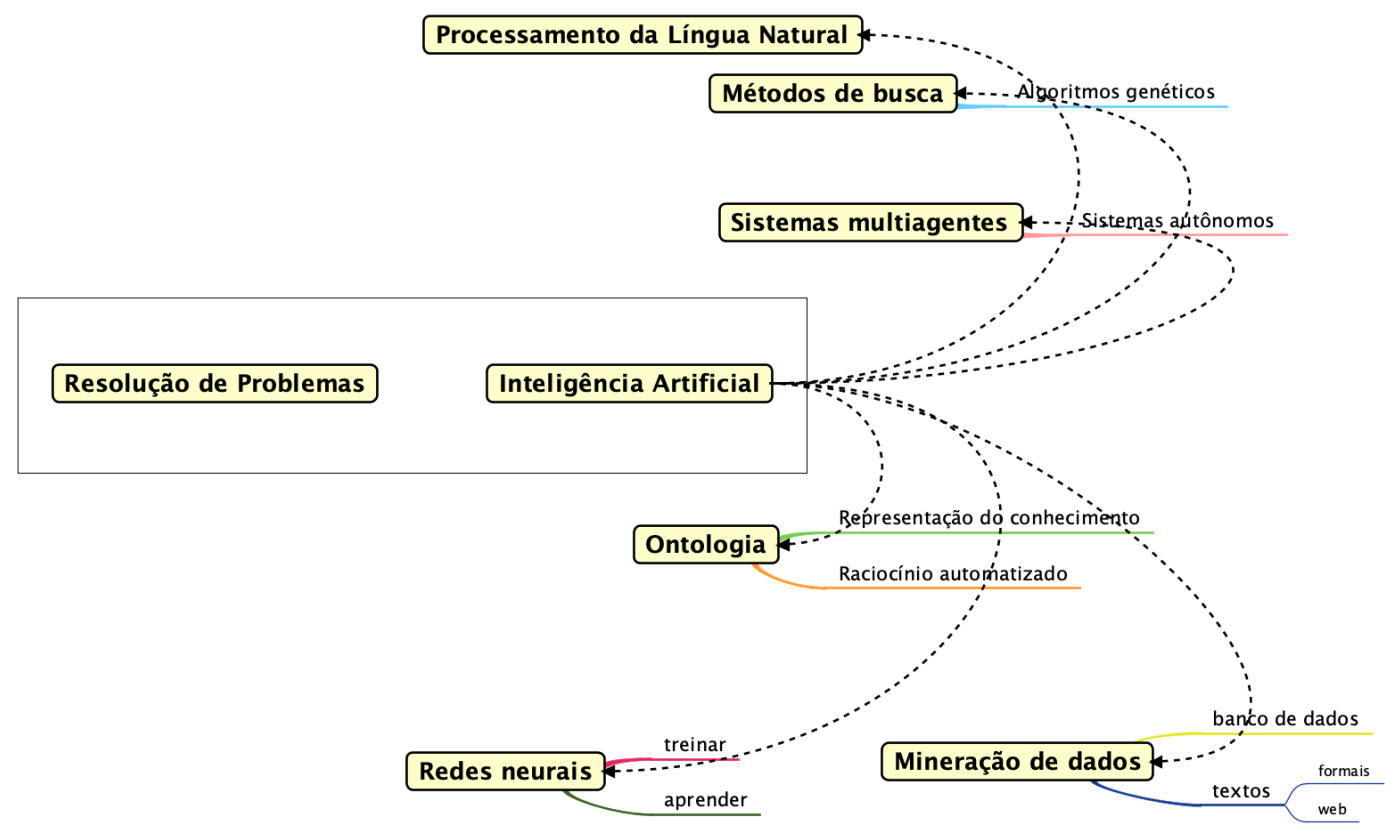

Figura 2 - Mapa mental da relação da Inteligência Artificial e suas subáreas. 
EDUGAÇÃO, SAÚDE

E TECNOLOGIA

26 A 28 DE OUTUBRO DE 2021

\section{QSOFN}

As aplicações da IA são diversas e, muitas vezes, inesperadas, surpreendendo consumidores, gestores, empresários e comunidades inteiras. Contudo, é possível afirmar, baseado pelo que se percebe no uso de sistemas de venda online de produtos/serviços, redes sociais, sistemas de streaming de música e vídeo, por exemplo, que o nicho dessas aplicações está em sistemas de recomendação (análise de perfil e padrões de comportamento), automação de tarefas, classificação e diagnóstico de textos, imagens, vídeos e áudios (Figura 3).

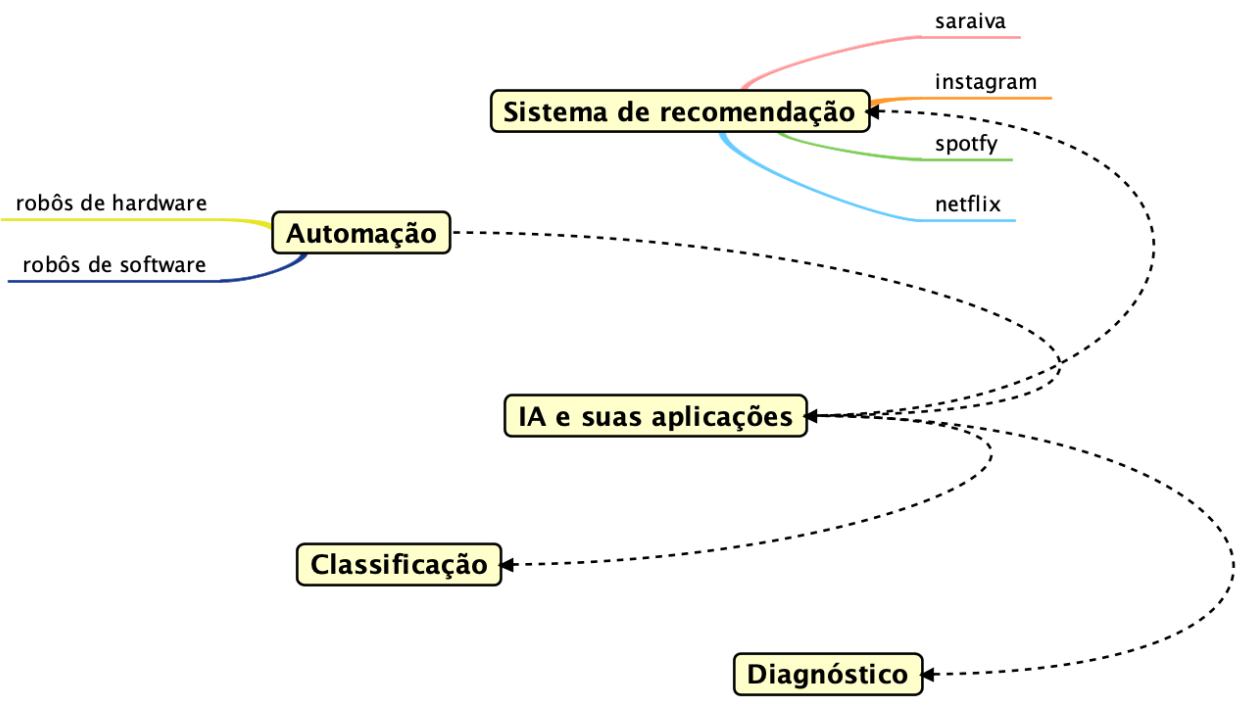

Figura 3 - Mapa mental da IA e suas possíveis aplicações.

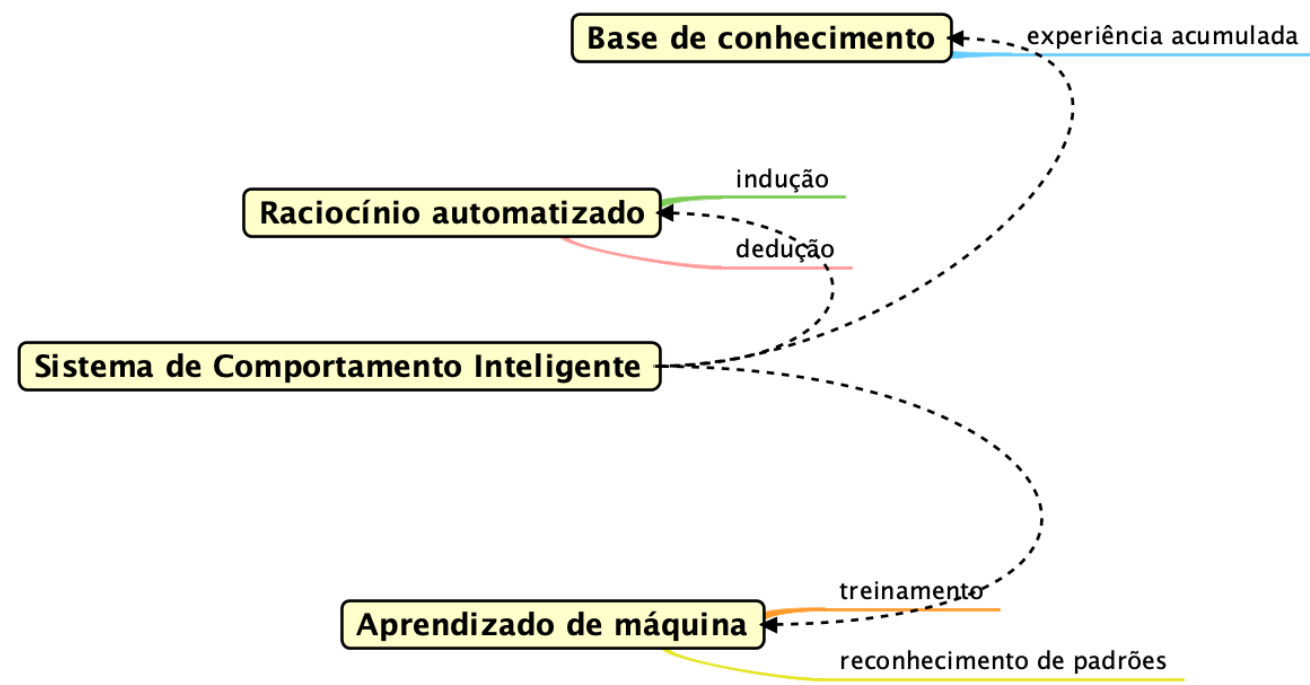

Figura 4 - Mapa mental dos elementos que formam o sistema de comportamento inteligente. 
Os sistemas projetados e construídos com técnicas de IA podem ser considerados como Sistemas de Comportamento Inteligente, como ilustra a Figura 4. Esses sistemas devem possuir: i) base de conhecimento (repositório de experiências ou situações ou casos, em formas de fatos e regras, acumulados em uma linha de tempo); ii) um motor de raciocínio automatizado, capaz de realizar deduções ou induções lógicas sobre as experiências armazenadas na base; iii) um recurso que por meio de treinamento repetitivo, o sistema reconheça padrões existentes na base de conhecimento, gerando assim, aprendizado de máquina.

Na Figura 5, então, é possível observar a relação das técnicas de IA no projeto e construção de Sistemas de Comportamento Inteligente, além de como as soluções deveriam tratar os problemas (estruturados ou não estruturados), por exemplo em uma área como Direito Ambiental.

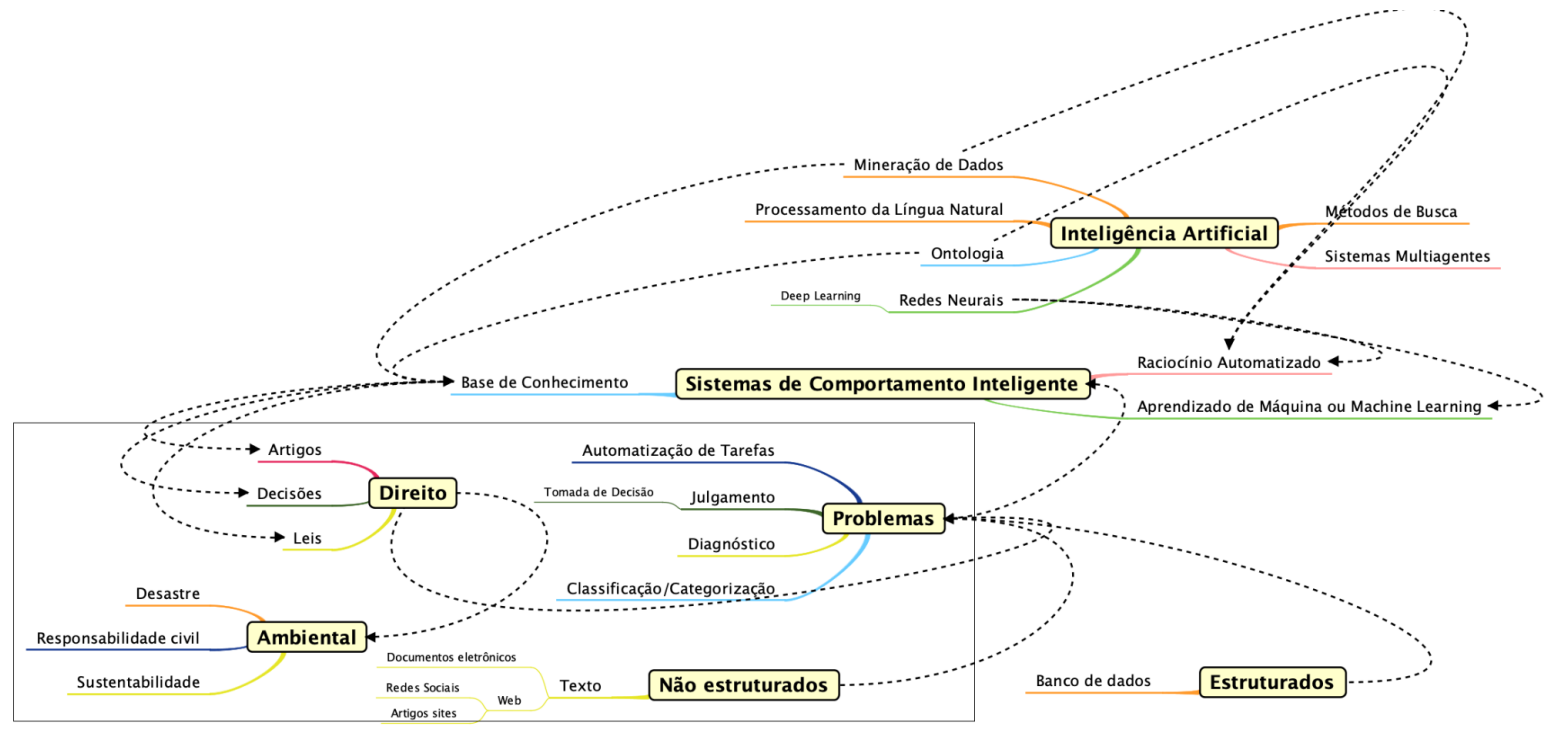

Figura 5 - Mapa mental de Sistemas de Comportamento Inteligente na área do Direito.

Por fim, na Figura 6, é possível observar a aplicação da técnica Ontologia para a construção de base de conhecimento no domínio do Direito, por exemplo. Uma vez criado esse modelo conceitual, que detalha conceitos e suas relações (de forma consensual, compartilhada e processável pelo computador), é possível aplicar mecanismos automatizados de raciocínio (reasoners) que são capazes de deduzir ou induzir logicamente sobre esse domínio. 


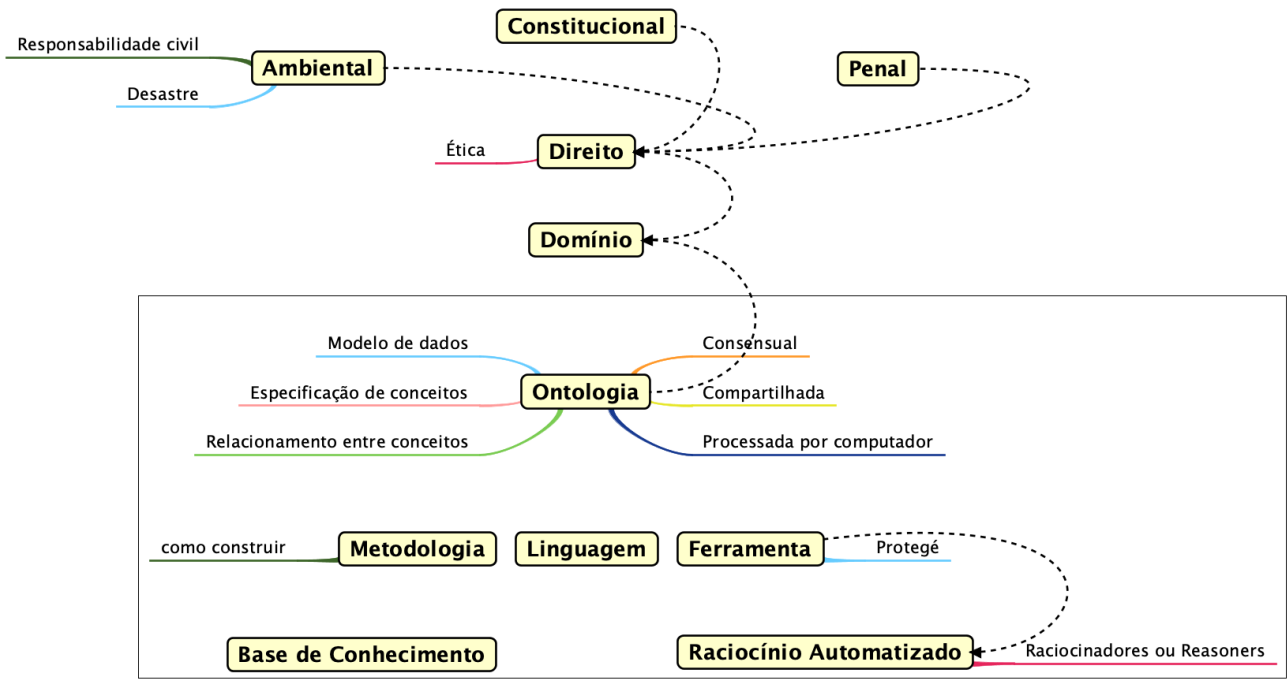

Figura 6 - Mapa mental de Ontologia, sua aplicação e seus elementos.

\section{METODOLOGIA, RESULTADOS E DISCUSSÕES}

A metodologia deste estudo (inicial) é baseada em revisão bibliográfica amparada por um estudo de caso, que busca modelar a dinâmica de um possível sistema com uso de representação de conhecimento e raciocínio automatizado via Ontologia.

Na Figura 7, encontra-se um Diagrama de Casos de Uso, que na Engenharia de Software, apresenta as principais funcionalidades de um sistema, como elas se relacionam e seus principais atores, neste caso, estudantes e advogados. A ideia do sistema é ser disponibilizado na Web, com acesso via software navegadores, em que usuários, previamente, cadastrados possam realizar pesquisas em bases jurídicas. Aqui, o sistema seria um concentrador de busca e de resultados, permitindo ao usuário armazenar os resultados de forma categorizada. Uma vez que o usuário dispara uma consulta, via palavras-chave ou expressões, o sistema também dispara consultas em revistas eletrônicas (doutrinas), em sites de tribunais (jurisprudência) e no site do Governo Federal - planalto.gov.br - (legislação). A relação de textos capturados ou selecionados são armazenados na base de dados desse sistema, que passa por um tratamento de limpeza dos textos (excluindo 
artigos, preposições, etc.). Em seguida, via uma representação de domínio (Ontologia) e uma ferramenta de raciocínio (reasoner), os textos da base são analisados com o objetivo de identificar e quantificar os acórdãos (decisão final proferida sobre um processo por tribunal superior, que funciona como paradigma para solucionar casos semelhantes) e os embasamentos jurídicos utilizados nesses textos, por exemplo.

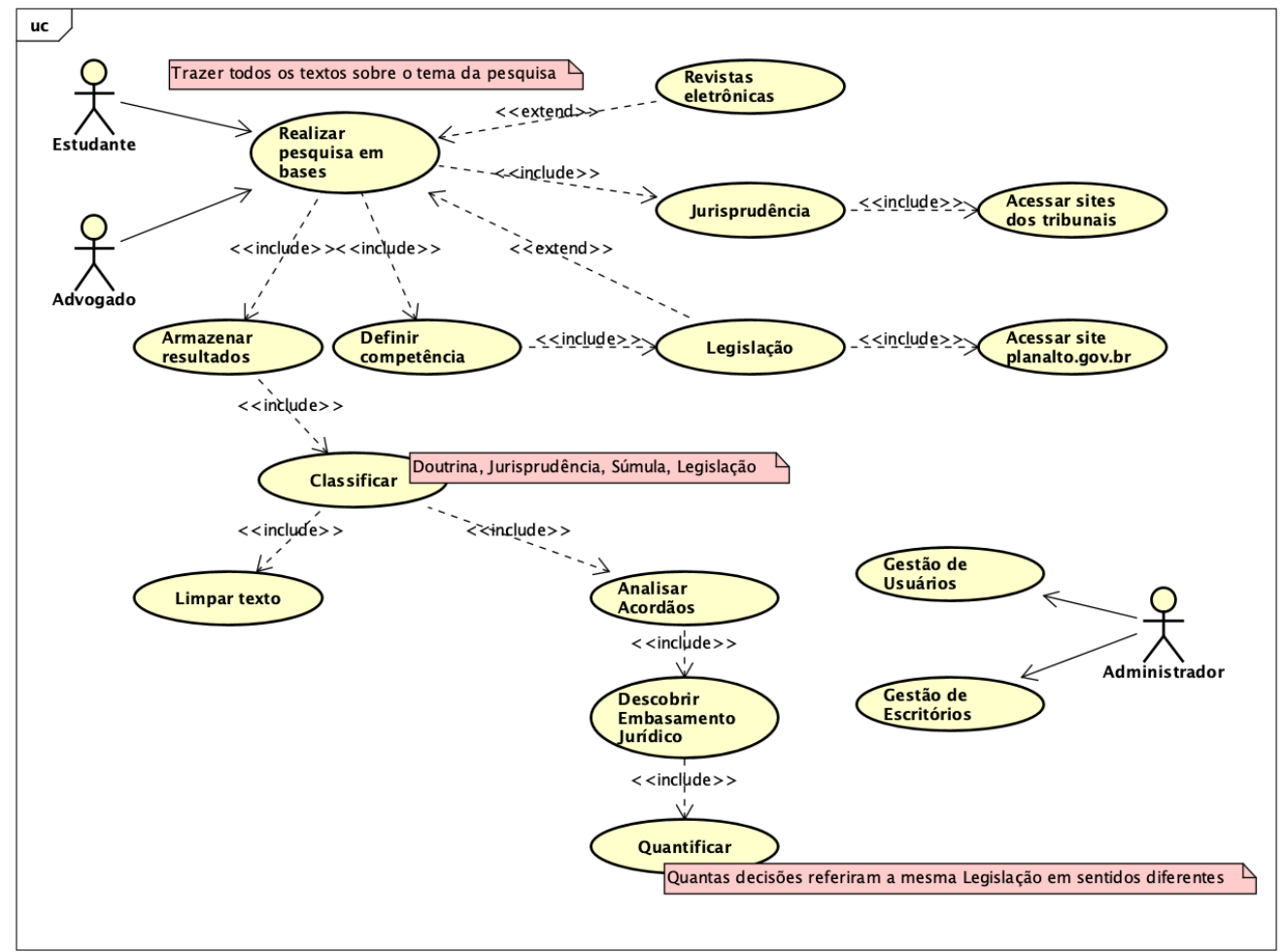

Figura 7 - Diagrama de casos de uso da proposta de um sistema online.

\section{CONCLUSÃO}

Ao longo do texto, tentou-se apresentar e discutir as principais técnicas de Inteligência Artificial e como são aplicadas em problemas estruturados e não estruturados, nos quais a área Jurídica encaixa-se. Ademais, buscou-se trazer Mapas Mentais para ilustrar os conceitos e suas relações. E nesse ponto, é possível fazer uma relação com Ontologia, ou seja, uma Mapa Mental é também uma representação formal de um domínio, que neste caso não é processada por 
máquina, pois não se gera seu código a partir dos diagramas, mas que ilustra adequadamente o conceito de Ontologia.

A Inteligência Artificial, com suas técnicas e aplicações, tem um foco claro no processo de compreender (e talvez substituir) o comportamento humano, que pode nos levar a situações negativas de monitoramento e controle, muitas vezes sem que tenhamos plena consciência dessa ingerência em nossa vida. Por isso, as discussões sobre o tema precisam ser mais profundas e difundidas, uma vez que a IA está presente em nosso dia a dia e veio para ficar. Ser parte ativa e interessada nessas discussões pode ser tanto uma estratégia de abertura de possibilidades de negócios como um mecanismo de proteção.

\section{REFERÊNCIAS}

GRUBER, Tom R. Toward principles for the design of ontologies used for

knowledge sharing. In International Journal of Human-Computer Studies, Vol. 43, Issues 4-5, November 1995, pp. 907-928. Disponível em

https://web.archive.org/web/20100715223053/http://tomgruber.org/writing/onto-desig n.htm. Acessado em Setembro de 2021.

RUSSELL, Stuart J.; NORVIG, Peter. Inteligência Artificial. Elsevier: Brasil, 2014. 